\title{
BMC Dermatology reviewer acknowledgement 2014
}

Hayley Henderson

\section{Contributing reviewers}

The editors of BMC Dermatology would like to thank all our reviewers who have contributed to the journal in Volume 14 (2014).

Ilknur Altunay
Turkey
Isabel Andia
Spain
Zoe Apalla
Greece
Alejandro Arenas-Pinto
UK
Giuseppe Argenziano
Italy
Wioletta Baranska-Rybak
Poland
Ioannis Bassukas
Greece
Robert Bransfield
USA
Robert Brodell
USA
Alex Burdorf
Netherlands
Ian Burgess
UK
Marco Castori
Italy
Hector Chinoy
UK

\author{
Elliot Coups \\ USA
}

Marian Currie

Australia

Soheil Dadras

USA

Robert Dellavalle

USA

Hoelzle Erhard

Germany

Stefano Fedele

UK

Steven Feldman

USA

Noriki Fujimoto

Japan

Georgios Gaitanis

Greece

Rebat Halder

USA

Pilar Iranzo

Spain

Takamichi Ito

Japan

Munenari Itoh

Japan
Feroze Kaliyadan

Saudi Arabia

Grazyna Kaminska-Winciorek

Poland

Jennifer Koplin

Australia

Chembolli Lakshmi

India

Peter Lio

USA

Marie Loden

Sweden

Ingrid Lundberg

Sweden

Eve Maubec

France

David Nelson

USA

Emiko Noguchi

Japan

Rei Ogawa

Japan

Anthony Ormerod

UK

Tae Hwan Park

Korea, South 
Stefano Piaserico

Italy

Andor Pivarcsi

Sweden

Gaelle Quereux

France

Kristian Reich

Germany

June Robinson

USA

Laura Sawyer

UK
Fabiana Schuelter-Trevisol

Brazil

Daniel Shin

USA

Gregory Sonnenberg

USA

H. Peter Soyer

Australia

Wim Stevens

Belgium

Makoto Sugaya

Japan
Michael Sweeney

USA

Brian Tait

Australia

Andrés Tirado-Sánchez Mexico

Hywel Williams

UK

Miriam Wittmann

UK

Xianyong Yin

China 